\title{
Experience of the Department of Surgery "A" Point-G Hospital in the Management of Hemorrhoids (Mali)
}

\section{Sidiki Keita ${ }^{*}$, Koniba Keita ${ }^{2}$, Mahamadou Coulibaly', Lamine Soumare', Moussa Sissoko', Oumar Sacko', Sekou Koumaré1, Adama K. Koita', Soumaîla Keita1, Zimogo Zié Sanogo'}

${ }^{1}$ General Surgery “A” Department, Hospital Point-G., Bamako, Mali

${ }^{2}$ General Surgery Department, Hospital BSS, Kati, Mali

Email: ^sidikibafing@yahoo.fr

How to cite this paper: Keita, S., Keita, K., Coulibaly, M., Soumare, L., Sissoko, M., Sacko, O., Koumaré, S., Koita, A.K., Keita, S. and Sanogo, Z.Z. (2020) Experience of the Department of Surgery "A" Point-G Hospital in the Management of Hemorrhoids (Mali). Surgical Science, 11, 435-445. https://doi.org/10.4236/ss.2020.1112045

Received: November 10, 2020 Accepted: December 15, 2020 Published: December 18, 2020

Copyright $\odot 2020$ by author(s) and Scientific Research Publishing Inc. This work is licensed under the Creative Commons Attribution International License (CC BY 4.0).

http://creativecommons.org/licenses/by/4.0/

(c) (i) Open Access

\begin{abstract}
Introduction: Hemorrhoidal disease is the most common condition in proctology: it is defined by signs or symptoms attributed to hemorrhoids. There is no parallel between the extent of hemorrhoidal anatomical disease and the symptoms described by patients. Our objective was to assess the incidence of hemorrhoidal disease, to diagnose it and to propose therapeutics. Patients and Method: This was a prospective and descriptive study based on a pre-established protocol that had taken place over a 12-month period. All patients (92 cases) underwent a general examination and a proctological examination. Anoscopy has often been associated with rectoscope with or without biopsy of the rectal mucosa. The inclusion criteria were the finding of hemorrhoidal disease, associated or not with other proctological diseases and the criteria for non-inclusion was any other anorectal pathology. Results: Among our patients there were 69 men or $75 \%$ of cases and 23 women or $25 \%$ of cases. The sex ratio was 3 in favor of men, the average age was 35.42 . The duration of progression of the disease was between 0 - 2 years in $59.78 \%$. Constipation (60 cases or $66.3 \%$ ) and diarrhea (60 cases or $22.8 \%$ ) were the factors that triggered the disease. Hemorrhoids with 3 packets were the most common (49 cases or $53.26 \%$ ). 56 patients were treated medically and 36 patients were operated on. The surgical technique was simple hemorrhoidectomy according to MILLIGAN and MORGAN. It was associated with a fissurectomy in $8.33 \%$ of cases, a fistulectomy in $13.90 \%$ of cases or a thrombectomy in $33.33 \%$. Early surgical sequels were dominated by pain in all patients, rectorragie, and urine retention. We did not deplore any deaths. Conclusion: Hemorrhoidal disease is an anatomoclinical entity that is still poorly elucidated and no direct link between the nature of the lesions and symptomatol-
\end{abstract}


ogy can be established.

\section{Keywords}

Proctology, Hemorrhoids, Management

\section{Introduction}

Hemorrhoidal disease is the most common condition in proctology and ranging between $4.4 \%$ and $36.4 \%$ of the general population [1]. No one has ever defined hemorrhoids; this problem has always irritated proctologists. This is due to the difficulty of differentiating the normal morphology of the anal canal from the pathological aspect, the strangeness of red hemorrhages in a venous disease, the discordance between the anatomopathological findings and the clinic [1]. Hemorrhoidal disease includes all the potential pathologies that can affect these hemorrhoids. Internal hemorrhoids can prolapse, bleed and rarely thrombose. There is no parallelism between the importance of hemorrhoidal anatomical disease and the symptoms described by patients. In other words, some suffer from small hemorrhoids that are not prolapsed and other patients have significant internal hemorrhoidal incidence without symptoms.

The pathogenesis of hemorrhoidal disease is based on ancient vascular and mechanical theories which are nevertheless the basis of the therapeutic approach (decrease in the vascular and inflammatory component when it exists) (Vascular theory) and strengthening of the mechanical means useful to maintain or reposition the hemorrhoid tissue in a favorable anatomical position (treatments and some surgical treatments). Medical treatment of hemorrhoidal disease uses topical treatments that are used in cases of acute manifestations, hygienic-dietary measures and transit regulators. Instrumental treatment is only for internal hemorrhoids that manifest themselves in rectorragie and/or prolapse. Its goal is to create scarring fibrosis that fixes the mucous membrane to the deep plane and reduces vascularization [2]. The literature suggests that hemorrhoid pedicular exeresis is the intervention of choice used by most authors [3] [4] [5]. Closed wound variants (Fergusson) or semi-closed (Parks) are favoured by North American authors; the open wound variant (Milligan and Morgan) to that of European authors, especially French ones. Circular stapled hemorrhoidopexy is a recent technique that does not perform a hemorrhoidectomy. It aims to correct muco-hemorrhoidal prolapse. The objective of this study was to assess the incidence of hemorrhoidal disease in our context, to diagnose it and to propose therapeutics based on the location and the annoying or not characteristic, while comparing our results with those made in this direction through a review of the literature.

\section{Patients and Method}

This was an observational, descriptive and prospective study according to a 
pre-established protocol that had taken place over 12 months (from 1 January 2017 to 31 December 2018). The study was carried out in the General Surgery Department of the "A" Hospital at Point "G" in Bamako. We had collected 92 patients. The 92 patients were interviewed and examined by the same doctor, in an identical pattern. Of these, 36 patients were operated on and 56 patients received medical treatment.

All patients underwent a general examination and a proctological examination. During this proctological examination the knee-pectoral position on a table was adopted. The rectal touch was systematic with a fingerer well lubricated by Vaseline.

The examination ended with an anoscopy, the anoscopy was often associated with rectoscopy with or without biopsy of the mucosa.

Preoperative assessment included: blood-rhesus, blood glucose, serum creatinine, prothrombin levels, activated cephalin time, blood formula count and platelet levels.

All 36 patients operated on were operated on by Milligan-Morgan's technique at Saint Mark's Hospital in London. The gynecological position was suitable. The patient is supine, thighs semi-flexed at right angles. Abduction should be limited to avoid impact on the spine. The buttocks extend slightly over the edge of the table. Two helpers are needed and the operator is seated opposite the patient.

Our inclusion criteria were: all patients admitted during the study period for hemorrhoids. The criteria for non-inclusion were: all other isolated anorectal pathologies and anorectal cancer pathologies.

Data extraction: Data extraction was done from texts and possibly tables and figures.

Parameters studied.

The following parameters: Were analyzed: patient demographic characteristics (age, gender), clinical presentation, diagnostic confirmation, methods and therapeutic results.

Statistical method: The data collected was put together in a database in the form of an Excel ${ }^{\circledR}$ table. Variables are expressed as mean or median with extremes. Data analysis was performed using Epi Info 7TM software.

\section{Results}

Of our 92 patients, 69 were men (75\%) and 23 were women (25\%) or a sex ratio of 3 men to a woman (Table 1). The average age was 35.42. Patients in a sitting position at work dominated this series with $56.52 \%$ of cases compared to $32.62 \%$ of patients occupying standing position. The duration of progression of the disease was $0-2$ years for 55 patients (59.78\%) and $3-6$ years for 30 patients $32.60 \%$ (Table 2). Constipation was responsible for the outbreak of the hemorrhoidal attack in 60 patients or (66.3\%) Cases diarrhea was responsible for $22.8 \%$ (Table 3). Pain at defecation was the most common call sign with $97.8 \%$, rectal bleeding was present in 57 patients (62\%), seeps were present in 28 patients 
Table 1. Distribution of patients by age and sex.

\begin{tabular}{|c|c|c|c|c|c|c|}
\hline \multirow{2}{*}{ Age } & \multicolumn{4}{|c|}{ Sex } & \multirow{2}{*}{ Total } & \multirow{2}{*}{ Percentage } \\
\hline & $\mathbf{M}$ & $\%$ & F & $\%$ & & \\
\hline 0 - 20 years & 1 & 1.45 & 2 & 8.70 & 3 & 3.26 \\
\hline 21 - 40 years & 48 & 69.57 & 16 & 69.56 & 64 & 69.58 \\
\hline 41 - 60 years & 20 & 28.98 & 4 & 17.39 & 24 & 26.09 \\
\hline$>60$ years & 0 & 0 & 1 & 4.35 & 1 & 1.07 \\
\hline Total & 69 & 100 & 23 & 100 & 92 & 100 \\
\hline
\end{tabular}

Table 2. Duration of development of disease.

\begin{tabular}{ccc}
\hline Duration of development & Number of cases & Percentage \\
\hline 0 - 2 years & 55 & 59.78 \\
$3-6$ years & 30 & 32.60 \\
7 - 10 years & 5 & 5.44 \\
$>10$ years & 2 & 2.18 \\
Total & 92 & 100 \\
\hline
\end{tabular}

Table 3. Triggering factors.

\begin{tabular}{ccc}
\hline Triggering factors & Number of cases & Percentage \\
\hline diarrhea & 21 & 22.8 \\
Constipation & 60 & 66.3 \\
Pregnancy & 5 & 5.4 \\
Menstruation & 3 & 3.3 \\
Alcohol & 3 & 2.2 \\
Total & 92 & 100 \\
\hline
\end{tabular}

(30.4\%). Several signs of call may exist in the same patient, the combination of swelling and pain in defecation was present 62 patients $(67.39 \%)$. Only 5 out of 92 patients were diagnosed with rectal examination. On the other hand, anoscopy made it possible to diagnose the disease in all patients: in 79 patients or (85.9\%) hemorrhoids were simple, in 5 patients they were associated with fistula; in 4 patients, they were associated with a crack and in 4 patients hemorrhoids were thrombosed. 3-pack hemorrhoids were more common with 49 cases or $53.26 \%$. The usual seat of hemorrhoidal packages was $3 \mathrm{H}, 7 \mathrm{H}, 11 \mathrm{H}$ depending on the hands of a watch or 49 cases (53.26\%). The stages found according to the SUDACA classification (Table 4) were: stage I (20 patients), stage II (39 patients), stage III (33 patients). The parasitological examination of stools found: Entamoeba histolytica (45 cases or 67.20\%), Oxyuroses (7 cases or 10.44\%), Candida albicans (10 cases or 14.92\%), Ascaris (5 cases or 7.47).

Medical treatment consisted of medical and hygienic management in 52 patients. There was no special preparation of the patient for surgery. To ensure the 
Table 4. Classification of Hemorrhoids according to the stages (SUDACA et Coll.) [12].

\begin{tabular}{ccc}
\hline Stages & Number of cases & Percentage \\
\hline I & 20 & 21.74 \\
II & 39 & 42.40 \\
III & 33 & 35.86 \\
Total & 92 & 100 \\
\hline
\end{tabular}

emptiness of the rectal bulb, we used micro-enemas with local laxatives on the eve of the procedure. The gynecological or so-called "size" position was adopted in all our patients. The patient is in back decubitus, buttocks overflowing the edge of the table, legs hanging from leggings. Patients were operated under general anesthesia in $86.1 \%$ and under locoregional anesthesia in $13.9 \%$ of cases. Surgical treatment was performed in 36 patients. All patients operated on were operated on by the technique of pedicular hemorrhoidectomy according to Milligan and Morgan of Saint Marks Hospital in London. The topography of the lesions shows a predominance of ligatures at three, seven o'clock and 11 o'clock. Hemorrhoidectomy was associated with either a cleft with 3 patients (8.33\%), a fistulectomy in 5 patients (13.90\%) thrombectomy in 12 patients (33.33\%). Pain evaluation between the first and seventh days of the operation found 4 cases of pain estimated at low intensity, rectorragie was considered minimal in 8 patients and urinary retention was present in 3 patients. The result of surgical treatment between $6^{\text {th }}$ week and $12^{\text {th }}$ week was considered satisfactory in 28 patients, 5 patients had anal narrowing and in 3 patients the anal canal was considered unsightly. The one-year evolution was considered satisfactory with the total disappearance of call signs in our 36 operated patients. Postoperative mortality in our series was zero. Anatomopathological examination of the operating rooms found no cancerous lesions.

\section{Discussion}

In hemorrhoidal disease, the predominance of the male sex was evoked. The distribution by sex was 3 men for 1 woman. In the female population, it appears that the most common hemorrhoidal disease is thrombosis (most often external). In studies that carried out a systematic review, it is reported in $8 \%, 24 \%$, $38 \%$ of parturients during the third trimester of pregnancy and in $12.2 \%, 20 \%$, and $34 \%$ of them in the immediate postpartum [6]. The age group most affected in this pathology was $21-40$ or $69.58 \%$ of the cases in our series, with an average age of $35.42-0.48$. The youngest patient was 6 years old. Hemorrhoidal pathology is very rare in children except in adolescence and in cases of portal hypertension. It should not be confused with the physiological localized venous dilations that can be observed between 2 and 5 years of age. These venous dilations are bluish, soft, painless, non-hemorrhagic, purely marginal. They do not prejudge the subsequent onset of a hemorrhoidal pathology [7]. Hemorrhoidal disease is a chronic disease that evolves in successive outbreaks, and patients do not 
consult early. The interrogation reveals that the first hemorrhoidal manifestations usually date back 4 years. Constipation, diarrhea (even caused by irritating laxatives) was responsible for triggering the hemorrhoidal attack. The role of childbirth seems to be paramount since postpartum thrombosis occurs in $91 \%$ of cases within 24 hours of delivery. It also appears that difficult deliveries with a tear in the small lips or a large baby are more often associated with thrombosis. In addition, there is much less thrombosis after caesarean deliveries than after vaginal tract (4\% versus $23 \%$ ) [6].

Pain at defecation was the frequent call sign and was present in 90 patients or $91.8 \%$. Hemorrhoidal pain is a weight with false need, feeling of intra-anal foreign body and incomplete exoneration [8]. Swelling, which is one of the main signs of hemorrhoidal disease, was present in $60 \%$ of cases [9]. It could be mobile, externalized during defecation and reduced spontaneously or by manual pressure. Swelling can be permanent, irreducible that can choke, thrombosis and become very painful. In our study, swelling was present in $77 \%$ of patients or 83.7\%. Rectora is the most representative symptom of hemorrhoids, since they owe their name to it. In our series, rectal bleeding was present in $57 \%$ of patients or $62 \%$ of cases. This was medium-sized hemorrhage, irregular reproduction, relatively well tolerated. It occurred during defecation was made of bright red blood. The combination of pruritus and oozing was present in $55.43 \%$ of patients. These symptoms are mainly favoured by anatomical conditions of the margin: maceration, skin folds, microbial density, inflammatory lesions [10] [11]. Rectal touch had little interest in the diagnosis of hemorrhoids, it allows above all to make the diagnosis of thrombosis, rectal dyschesia, to appreciate the tone of the anal sphincter, to look for other pathology such as cancer of the anus or rectum. On the other hand, anoscopy is an indispensable examination in pathology, and allows to assess the general condition of the mucosa, the volume and the seat of the various hemorrhoidal packages, the associated lesions. Anoscopy has allowed the classification to be classified into three stages for therapeutic purposes [12].

Medical treatment aims to eliminate functional manifestations related to hemorrhoids; it is therefore not intended to suppress an anatomical situation, as hemorrhoids are a normally present anatomical structure. We used medical treatment in 56 patients ( $60.86 \%$ of cases) who suffered from stage I and stage II simple hemorrhoids not associated with fistula or fissure or major rectal bleeding. This treatment included: hygienic-dietary rules: drinking large amounts of water, increasing the fecal bowl by absorption, green vegetables, fresh and dried fruit, wholemeal bread or bran bread, wheat bran, local treatments: use of cold, seat baths; local anaesthetics, topical with a veinotonic or prokinetic. No recommendations can be made for local treatments: use of cold, seat baths, local anesthetics, topicals with a prokinetic or veinotonic. A controlled study showed the absence of harmfulness and a $90 \%$ benefit in patients with hemorrhoid symptoms treated with local topicals. The lack of long-term benefit and demon- 
strated preventive action on new outbreaks does not allow for any advice on their prolonged use [6]. Transit regulation is recommended in case of symptoms related to internal and external hemorrhoidal disease, both curative and preventive. It is the only long-term preventive measure with demonstrated efficacy on hemorrhoidal symptoms. The use of phlebotropes is based on the pathophysiological concept involving the vascular component of hemorrhoids: diosmin, troxerutin, Ginkgo biloba derivatives, hydroxyethyl rutoside. Two recent meta-analyses have shown a benefit to the use of veinotropic for pruritus, rectal bleeding and oozing [10]. Nonsteroidal anti-inflammatory drugs are effective on pains of internal or external hemorrhoidal thrombosis, they can be prescribed in combination with laxatives. Data on the use of corticosteroids are not available in general. Peripheral painkillers (paracetamol, dextropropoxyphene) are effective. In pregnant or lactating women, it is advisable to correct transit disorders often associated with, authorized painkillers (paracetamol) can be prescribed as a short cure [6] [10]. In the case of external or internal hemorrhoid thrombosis, per bone corticosteroids may be used in a short cure, but not nonsteroidal anti-inflammatory drugs (NSAIDs). INSNs should be avoided from the beginning of pregnancy and are formally constrained from the $6^{\text {th }}$ month (24SA) until delivery. They can be used during breastfeeding [6] [10]. In our series 56 patients (60.86\% of cases) were treated exclusively medically and especially stage I and stage II hemorrhoids. The results were satisfactory, to a week a noticeable improvement of the various call signs: pain disappeared in $82.14 \%$ of cases, rectal bleeding in $32.14 \%$ and anal pruritus in $57.1 \%$. Between the $4^{\text {th }}$ and $6^{\text {th }}$ week there was a disappearance of the signs of appeal. Among the 36 patients operated 6 patients who suffered from stage II and stage III hemorrhoidal disease with thrombosis and pain were by the medical method based on analgesics, nonsteroidal anti-inflammatory drugs and veinotrope prior to the intervention. This treatment allowed a good preparation for surgery.

Instrumental treatment is only for internal hemorrhoids that manifest themselves in rectal bleeding and/or prolapse. Its goal is to create scarring fibrosis that fixes the mucous membrane to the deep plane and reduces vascularization. Many techniques have been described, the three instrumental treatments validated in the literature are: infrared clotting photo; sclerosis injections and elastic ligatures [2] [6] [13]. Complications of instrumental treatment must be known. This is pain to type of discomfort, intra anal foreign body sensation, rectorragie and severe complications. Pains are reported in 20\% - 35\% after photo infrared clotting, in $9 \%$ to $70 \%$ after sclerosis injection, $5 \%$ to $85 \%$ after elastic ligation [2]. Rectora associated with superficial mucous necrosis is possible until the tenth day. They are observed in $2 \%$ to $10 \%$ of cases after sclerosis injection, $1 \%$ to $15 \%$ sclerosis ligation and $5 \%$ to $25 \%$ after infrared clotting. Ligation can lead to external and/or internal hemorrhoidal thrombosis, dysuria or major algia in $1 \%$ to $5 \%$ of cases. Severe hemorrhagic or infectious complications are exceptional but need to be known quickly. Massive hemorrhages on bedsores occur- 
ring between the fifth and $12^{\text {th }}$ day require surgical hemostasis. They are mainly caused by elastic ligatures ( $0.5 \%$ to $2 \%$ of cases) but mono-polar or bipolar clotting accounts for 0 to $8 \%$ of cases. Five cases of pelvic or genitourinary suppurations have been reported after sclerosis injection. Fifteen cases of pelvic cellulite including 6 deaths have been reported elastic ligation [2]. The effectiveness of instrumental treatments is comparable to 3 months, regardless of the technique used (improvement of symptoms in $70 \%$ to $90 \%$ of cases) [2]. At one year, the positive results of sclerosis injections and infrared photo clotting are only $50 \%$ [2]. At a distance, at three years, elastic ligation is the most effective technique (75\% to $90 \%)$.

Surgical treatment of hemorrhoids is indicated in about $10 \%$ of patients [3] [5]. The literature shows that the pedicular exeresis of hemorrhoids is the intervention of choice. Closed wound variants (Fergusson) or semi-closed wounds (Parks) are favoured by North American authors; the open wound variant (Milligan and Morgan) has that of European authors in particular French. Longo's circular stapled hemorrhoidopexia (or anopexia) is a technique that does not perform a hemorrhoidectomy. It aims to correct muco-hemorrhoidal prolapse. The Guided Doppler ligation of hemorrhoidal arteries, although performed under anesthesia, this technique can be compared to instrumental treatments because it is ambulatory and does not require tissue exeresis. It performs the ligation of the hemorrhoidal arteries after spotting them with a doppler carried by a windowed anoscope. Arterial ligation alone is not recommended for grade 4. The combination of mucopexia and arterial ligation gives better results. The overall efficiency is $92 \%$ at one year and $73 \%$ at 5 years. In case of associated mucopexia the overall efficacy is $91 \%$ at 15 months and $88 \%$ at 36 months [3]. In our study the patients did not receive any special preparation. To ensure the emptiness of the rectal bulb, we used micro-enemas. The gynecological or so-called "size" position was adopted in all our patients: 36 cases. The patient is in back decubitus, knees arrowed on the chest, legs hanging from leggings, buttocks overflowing the edge of the table. General anesthesia was used in 31 patients, or $86.1 \%$ of cases, and 5 patients or $13.9 \%$ of patients were operated on under locoregional anesthesia. The surgical technique used in our study was separate package pedicle hemorrhoidectomy or the saint MARK Hospital technique in London. Immediate postoperative after-operative procedures, from the first day to the $7^{\text {th }}$ day after the procedure, were marked by pain, usually as soon as the pain woke up, was alleviated, especially when the "compress pudding" was removed from the anal canal at the end of the procedure. This ointment-soaked compressed pudding prevented the adhesions of the surgical wounds that favoured the occurrence of stenosis and, on the other hand, to stop the bleeding in the tablecloth. In 31 patients, or $86.1 \%$ of cases, the pain was acceptable, well tolerated leaving no painful memory and there was no need to prescribe painkillers. In 4 patients or $11.1 \%$ of cases a painful background persisted for the first 3 days after hemorrhoidectomy especially at the time of the first stool. In one case 
$2.8 \%$ the pain was severe after the stool and it lasted 5 days. DAVY A [14] found immediate postoperative pain acceptable and well tolerated in $2 / 3$ of his patients (n-900). The pain was severe in $1 / 3$ of the cases. It was pain at defecation. SUDUCA et al. [12] noted that $12 \%$ of patients had no pain, $63 \%$ experienced moderate pain and $6 \%$ experienced severe pain (n-1000). Of the painful consequences, $40 \%$ were calmed by oral painkillers and $60 \%$ by parenteral means. Rectal bleeding is a serious complication of hemorrhoidectomy [2]. In our study, 8 cases were observed, i.e. $22.2 \%$ of minimal rectal bleeding, and did not require revision in the operating room. SOULLARD [1] reported 3 cases of true rectal bleeding $(n=100)$ during the first 24 hours. The extent of the bleeding forced him to return to the operating room. Correct and high ligation of the vascular pedicles avoided this complication [10]. They nevertheless deplored 1 case of serious hemorrhage in the postoperative period. Urine retention is a not uncommon complication after surgical treatment of hemorrhoids, it occurs in $15 \%$ of patients [3]. It requires an evacuator sounding in less than $10 \%$ of cases [3]. Their frequency is related to the volume infused during the procedure $(>1000 \mathrm{ml})$, the use of a long-acting local anesthetic or high doses of morphine, caudal anesthesia, multi-pedicular hemorrhoidectomy, intra-channeling and at an age of 40 to 50 years [3] [15] [16]. In our series we deplored 3 cases of urine retention or $8.34 \%$ of cases, this urine retention was lifted spontaneously. In our study 33 patients operated on or $91.66 \%$ of cases were reviewed in consultation one year after the surgery. No recurrence cases were reported, all of our patients rated the excellent method as a means of treating hemorrhoids. The length of hospitalization after this surgery was one week in literature [1] [17].

\section{Conclusion}

Hemorrhoidal disease is the most common condition encountered in proctology; it is defined by signs or symptoms attributed to hemorrhoids. There is no parallel between the extent of anatomical hemorrhoidal disease and the symptoms described by patients. The pathogenesis of hemorrhoidal disease is based on ancient vascular and mechanical theories which are nevertheless the basis of the therapeutic approach which is based on hygieno-dietary measures, the instrumental approach and surgical treatment. Pedicle hemorrhoidectomy according to the Milligan and Morgan technique, described at Saint Mark's Hospital in London in 1937, remains a widely practiced procedure. It has indeed specific indications where its results are excellent in internal hemorrhoids completely prolapsed (grade IV of Goligher), hypertrophic marisques, hemorrhoidal pathologies associated with another proctological pathology to be operated on (fissure, suppuration, condyloma, for example. example). In pregnant women, surgery should remain an exception to the failure of well-conducted medical treatment.

\section{The Limits of the Study}

The sample size was small, due to the duration of the study which was 12 
months and the incomplete records which could not be used. At one year, many patients followed up after surgery were lost to follow-up.

\section{Conflicts of Interest}

The authors declare no conflicts of interest regarding the publication of this paper.

\section{References}

[1] Soulard, J. and Contou, J.F. (1979) La ligature élastique: Procédé de traitement médical ambulatoire des hémorroïdes. Nouvelle Presse Médicale, 8, 1981-1982.

[2] Coulon, P. (2009) Instrumental Treatment of Hemorrhoidal. Gastroenterologie Clinique et Biologique, 33, 20-26. https://doi.org/10.1016/j.gcb.2009.07.030

[3] Pillant Le Moult, H., Aubert, M. and De Parades, V. (2015) Classical Treatment of Hemorrhoids. Journal of Visceral Surgery, 152, S3-S9. https://doi.org/10.1016/j.jviscsurg.2014.09.012

[4] Slim, K. and Gravié, J.F. (2003) What Surgical Technique for Stage IV Hemorrhoids? Which Surgical Technique for Stage IV Haemorrhoids? Annals of Surgery, 128, 616-618. https://doi.org/10.1016/j.anchir.2003.09.013

[5] De Parades, V.C., Parisot, C. and Atenza, P. (2005) L'hémorroïdectomie selon la technique de Milligan et Morgan. Journal de Chirurgie, 142, 302-305.

https://doi.org/10.1016/S0021-7697(05)80934-2

[6] Abramowitz, L. (2008) Management of Hemorrhoid Disease in the Pregnant Women. Biological Gastroenterology, 32, S210-S214.

https://doi.org/10.1016/j.gcb.2008.04.012

[7] Senéjoux, A. (2011) Proctologie pédiatrique courante. La Lettre de l'Hépato-gastroentérologue, 14.

[8] Contou, J.F. (1999) Complications of hemorroides. Revue de Médecine, 20, 1199-1201.

[9] Dembele, K. (1994) Clinical and Epidemiological Aspects of Diseases in Mali. Thesis Medicine, Bamako.

[10] Abramowitz, L., et al. (2001) Recommendations for Clinical Practice on the Treatment of Hemorrhoidal Disease. Gastroentérologie Clinique et Biologique, 25, 674-702.

[11] François, P. (2011) Treatment of Hemorrhoids from Theory to Practice. Hepato-Gastro and Digestive Oncology, 18, 203-206.

[12] Suduca, P. and Suduca, J.M. (1990) Hemorrhoids. EMC, Technical Edit, Paris, Stomach-Intestine, 9086 A105, 12-20.

[13] Nguyen, V., Jarry, J., Imperato, M., Farthouat, P., Michel, P. and Faucheron, J.L. (2012) French Experience in the Management of Hemorrhoids by HALTM Doppler-08/12/12. Journal of Visceral Surgery, 149, 412-416. https://doi.org/10.1016/j.jviscsurg.2012.10.004

[14] Davy, A. and Marel, U. (1971) Etudes des suites opératoires des hémorroïdectomies réalisées par la technique de l'hôpital Saint Mark de Londres: (à propos d'une série de 900 cas). An Chir, 97, 387-393.

[15] Shafik, A. (1983) Role of Warm Water in Inducing in Post Operative Urinary Retention after Anorectal Operations. Urologia Internationalis, 50, 213-217. https://doi.org/10.1159/000282487 
[16] Mariko, H. (1996) Hemorrhoid Study in "B" Surgery at the National Hospital of Point "G": About 152 Cases. Medical Thesis, Bamako.

[17] Sigrid, S. (1980) Results of Hemorrhoïdectomy According to Milligan: A Follow-Up Study of 100 Patients. Acta Chirurgica Scandinavica, 124, 444-453. 